\title{
TU/e EmonOWEN

\section{A Convex Optimization Framework for Minimum Lap Time Design and Control of Electric Race Cars}

\section{Citation for published version (APA):}

Borsboom, O. J. T., Fahdzyana, C. A., Hofman, T., \& Salazar, M. (2021). A Convex Optimization Framework for Minimum Lap Time Design and Control of Electric Race Cars. IEEE Transactions on Vehicular Technology, 70(9), 8478-8489. https://doi.org/10.1109/TVT.2021.3093164

DOI:

10.1109/TVT.2021.3093164

Document status and date:

Published: 01/09/2021

\section{Document Version:}

Accepted manuscript including changes made at the peer-review stage

\section{Please check the document version of this publication:}

- A submitted manuscript is the version of the article upon submission and before peer-review. There can be important differences between the submitted version and the official published version of record. People interested in the research are advised to contact the author for the final version of the publication, or visit the $\mathrm{DOI}$ to the publisher's website.

- The final author version and the galley proof are versions of the publication after peer review.

- The final published version features the final layout of the paper including the volume, issue and page numbers.

Link to publication

\section{General rights}

Copyright and moral rights for the publications made accessible in the public portal are retained by the authors and/or other copyright owners and it is a condition of accessing publications that users recognise and abide by the legal requirements associated with these rights.

- Users may download and print one copy of any publication from the public portal for the purpose of private study or research.

- You may not further distribute the material or use it for any profit-making activity or commercial gain

- You may freely distribute the URL identifying the publication in the public portal.

If the publication is distributed under the terms of Article 25fa of the Dutch Copyright Act, indicated by the "Taverne" license above, please follow below link for the End User Agreement:

www.tue.nl/taverne

Take down policy

If you believe that this document breaches copyright please contact us at:

openaccess@tue.nl

providing details and we will investigate your claim. 


\title{
A Convex Optimization Framework for Minimum Lap Time Design and Control of Electric Race Cars
}

\author{
Olaf Borsboom, Chyannie A. Fahdzyana, Theo Hofman, Mauro Salazar
}

\begin{abstract}
Lap time is the most important performance indicator in electric racing. In order to minimize it, the powertrain of the race car must be carefully designed and the energy carried on-board must be meticulously administered. In particular, to obtain the fastest possible lap time, it is important to select the components' technology and sizing accounting for the energy available on-board and for the resulting mass of the car. To facilitate efficient design, we present a convex optimization framework in this paper, to rapidly compute the minimum-lap-time control strategies for a battery electric race car. We first identify a convex model of the electric powertrain, including the battery, the electric motor, and two transmission technologies: a fixed-gear transmission (FGT) and a continuously variable transmission (CVT). Second, assuming an expert driver, we formulate the minimum-lap-time control problem in a convex fashion for a given driving path, and compute its globally optimal solution with second-order conic programming algorithms. Third, we showcase our framework on the Le Mans track by comparing the performance achievable with an FGT and a CVT, and validate it with nonlinear simulations. Our results show that for the given setup a CVT can balance its lower efficiency and higher weight with a higher-efficiency and more aggressive motor operation, significantly outperforming the lap time achievable with an FGT. Finally, we leverage the computational efficiency of our framework to carry out parameter studies on the components, revealing that optimizing the size of the battery and the motor for the specific scenario can considerably improve the achievable lap time, and that the best transmission strongly depends on the sizing decisions.
\end{abstract}

Index Terms-Convex optimization, time-optimal control, electric vehicles, powertrain design, racing

\section{INTRODUCTION}

$\mathrm{O}$ VER the last few years, the electrification of vehicle powertrain systems has gained significant interest. This trend is not only apparent in commercial and passenger vehicles, but also in the racing community, with the introduction of the fully-electric Formula E racing class and the strong hybridization of the Formula 1 (F1) power unit taking place in 2014 [1]. In contrast to electric commercial vehicles and heavy-duty trucks where the design and control goals are aimed at improving the cost, weight, range, and energy consumption, the most important performance indicator for battery electric race cars is the lap time. In a context where time differences are minimal, the control strategies managing the energy deployment of the vehicle on the racetrack as well as the design of its powertrain components have a significant impact on the achievable lap time and must therefore be carefully optimized. This challenge calls for methods to compute the minimum-lap-time control

The authors are with the Control Systems Technology group, Eindhoven University of Technology (TU/e), Eindhoven, $5600 \mathrm{MB}$, The Netherlands (e-mail: o.j.t.borsboometue.nl).

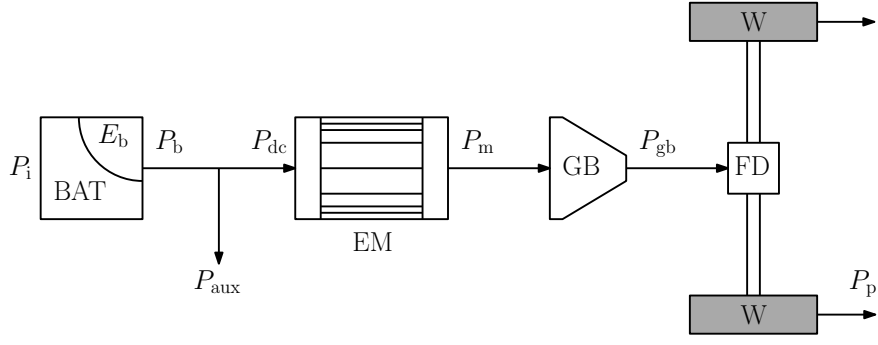

Fig. 1. Schematic layout of the considered electric race car powertrain consisting of a battery pack (BAT), an electric machine (EM), a transmission (GB), that can be either a fixed-gear transmission (FGT) or a continuously variable transmission (CVT), and a final drive reduction gear (FD) connected to the wheels $(\mathrm{W})$. The arrows indicate the power flows between the components.

strategies and concurrently conduct optimal powertrain design studies for battery electric race cars.

To efficiently compute the minimum-lap-time control strategies for the battery electric race car shown in Fig. 1, this paper presents a convex modeling and optimization framework. In addition, this framework allows to characterize the impact of powertrain design choices on the achievable lap time.

Related literature: The minimum-lap-time design and control problem studied in this paper pertains to three main research streams. The first line consists of offline control strategies for the energy management of hybrid electric vehicles. In general, such non-causal approaches are based on Pontryagin's Minimum Principle (PMP) [2]-[5], dynamic programming (DP) [6]-[8], and convex optimization [9][12], sometimes combining the three methods together [13], [14]. In addition, nonlinear optimization solvers are used for solving the hybrid electric energy management problem, such as IPOPT [15]. However, these methodologies minimize fuel-consumption and are often applicable to pre-determined driving cycles. Moreover, nonlinear optimization methods do not guarantee global optimality of the solution, nor guarantee convergence and have a relatively high computation time. The second research line is related to the time-optimal control of racing vehicles such as the hybrid electric F1 car. The majority of prior research in this field optimizes the velocity profile and driving trajectory simultaneously, using nonlinear optimization [16]-[21], or separately solve the energy management problem offline [22]-[25], using convex optimization. Yet these approaches do not capture the impact of the transmission on the motor or engine performance and hence the lap time. In electric vehicle powertrains, the choice of the transmission technology can have an enormous impact [26]. A continuously variable transmission (CVT) has the potential of allowing 
the electric motor to operate at the optimal operating points, while having the drawbacks of a relatively high mass and low efficiency [27]. These features would render a CVT an interesting transmission technology to be compared with a fixed-gear transmission (FGT) which, in contrast, is lighter and more efficient, but does not allow to control the EM operation. The third line of research comprises optimal sizing of hybrid or battery electric vehicles. This sizing is typically based on either high-fidelity simulation models combined with derivative-free algorithms [28]-[31], nonlinear methods [32][35] and convex optimization [36]-[39], which have mainly been used in passenger or commercial applications. Even though nonlinear solvers were employed for optimal design and control in racing scenarios [40], [41], they still have the aforementioned drawbacks regarding global optimality, convergence and computation time.

In conclusion, to the best of the authors' knowledge, the challenge of jointly optimizing the design and control of the full powertrain to minimize the lap time of a battery electric race car in a computationally-efficient manner and with global optimality guarantees has not been addressed yet. What is more, models to simultaneously optimize the torque, speed and transmission ratio of an electric drivetrain jointly with the speed profile in a convex fashion have not been presented to date.

Statement of contributions: To address this challenge, this paper presents a computationally-efficient optimization framework for battery electric race cars equipped with two types of transmissions, namely, a FGT or a CVT, whereby we optimize the design of the FGT, and the design and control of the CVT. In order to achieve this, we first derive a convex model of the powertrain shown in Fig. 1. Second, we formulate the minimum-lap-time control problem and solve it via secondorder conic programming. Third, we showcase our convex framework with case studies comparing the performance of different transmission technologies and component sizes on the Le Mans race track. A preliminary version of this paper was presented at the 2020 IEEE Vehicular Power and Propulsion Conference [42]. In this extended version, we identify a CVT model that explicitly captures the dependence of its losses on input speed, output speed, and input power. Moreover, we reframe the minimum-lap-time control problem in a fully convex form that no longer relies on iterative or nonlinear solution algorithms, but can be rapidly solved and provides global optimality guarantees. Finally, we validate our proposed models with nonlinear simulations and showcase our algorithmic framework via an extensive design case study on the motor and battery sizing.

Organization: The remainder of this paper is structured as follows: Section II presents the convex powertrain component modeling methods and the minimum-lap-time control problem. Section III presents numerical results and design studies for both an FGT and a CVT-equipped powertrain on the Le Mans track. Conclusions are drawn in Section IV, together with an outlook on future research.

\section{Methodology}

In order to compute the achievable lap time, we need to define a minimum-lap-time control problem and express the system dynamics and powertrain components as convex constraints. In this section, we identify a convex model of the race car shown in Fig. 1 and its powertrain and formulate the minimum-lap-time control problem, including the sizing of the motor, the battery and the transmission. The time-optimal control problem objective and domain definition are inspired by the methods applied to Formula 1 in [22], but differ in terms of powertrain topology and the explicit characterization of the transmission technology and its impact on the powertrain performance.

Fig. 1 shows a schematic layout of the powertrain of the vehicle under consideration. It is propelled by an electric motor (EM), which converts the electric energy from the battery pack to mechanical energy, considering both an FGT and a CVT as the transmission. Additionally, we also have a final reduction gear that transfers the resulting mechanical energy to the wheels. The input variables are the motor power $P_{\mathrm{m}}$ and, in the case of the CVT-equipped powertrain, also the transmission ratio $\gamma$. We treat the transmission ratio as a design variable when considering the FGT, and the ratio limitations $\gamma_{\min }$ and $\gamma_{\max }$ for the CVT. The state variables are the kinetic energy of the vehicle $E_{\text {kin }}$ and the amount of battery energy used since the start of the lap $E_{\mathrm{b}}$. The remaining power flows are the battery terminal power $P_{\mathrm{b}}$, the auxiliary power $P_{\text {aux }}$, the electrical EM power $P_{\mathrm{dc}}$, the transmission mechanical output power $P_{\mathrm{gb}}$, and the propulsion power $P_{\mathrm{p}}$. The powertrain controller cannot directly influence the driving path, which is rather set by the expert driver's steering input, hence we condense the $3 \mathrm{D}$ characteristics of the racetrack and the driven path in a $1 \mathrm{D}$ maximum-velocity profile $v_{\max }(s)$ depending on the longitudinal position of the car on the racetrack $s$. This profile can either be measured or pre-computed, and is usually not significantly affected by the energy management strategies [20], [21], but rather by the total mass of the car.

\section{A. Objective}

To construct the minimum-lap-time control problem, we begin by formulating its objective. We define the problem for one free-flow race lap in space domain. This way, we can directly implement position-dependent parameters such as the maximum velocity profile, and the problem has a finite horizon. The objective is to minimize the lap time $T$, i.e.,

$$
\min T=\min \int_{0}^{S} \frac{\mathrm{d} t}{\mathrm{~d} s}(s) \mathrm{d} s,
$$

where $S$ is the length of the track and $\frac{\mathrm{d} t}{\mathrm{~d} s}(s)$ is the lethargy, which can be interpreted as the time per driven distance, and is the inverse of the vehicle's velocity $v(s)$ :

$$
\frac{\mathrm{d} t}{\mathrm{~d} s}(s)=\frac{1}{v(s)} .
$$

However, since $\frac{\mathrm{d} t}{\mathrm{~d} s}(s)$ and $v(s)$ are both optimization variables, (2) is not convex. Re-arranging and relaxing (2) results in

$$
\frac{\mathrm{d} t}{\mathrm{~d} s}(s) \cdot v(s) \geq 1
$$


which is a geometric mean expression and can be written as the second-order conic constraint:

$$
\frac{\mathrm{d} t}{\mathrm{~d} s}(s) \cdot v_{0}+v(s) \cdot \frac{1}{v_{0}} \geq\left\|\frac{\mathrm{d} t}{\mathrm{~d} s}(s) \cdot v_{0}-v(s) \cdot \frac{1}{v_{0}}\right\|_{2},
$$

where $v_{0}=1 \mathrm{~m} / \mathrm{s}$ is a normalization term. Since from the objective (1) it is optimal to minimize the lethargy $\frac{\mathrm{d} t}{\mathrm{~d} s}(s)$, the solver will converge to a solution where constraint (3) holds with equality [22]. This and the following relaxations can be verified in post-processing, as will be done in Section III-B.

In the following sections, the longitudinal and powertrain dynamics and constraints will be expressed in space-domain and relaxed to a convex form whenever necessary. Since the space-derivative of energy is force, we will ultimately define the model of the powertrain in terms of forces. Thereby, power and force are related as

$$
F=\frac{P}{v}=P \cdot \frac{\mathrm{d} t}{\mathrm{~d} s},
$$

which can be used in post-processing to compute the optimal power $P^{\star}$ from the optimal force $F^{\star}$ and velocity $v^{\star}$.

\section{B. Longitudinal Vehicle Dynamics}

To extend the minimum-lap-time control problem, this section derives the longitudinal vehicle dynamics and expresses them in a convex form in space domain. Starting from the wheels and following the powertrain upstream, we subsequently express the components as constraints to our problem, in this and the following subsections.

In order to connect the vehicle dynamics and the objective, we define the physical constraint on the kinetic energy

$$
E_{\mathrm{kin}}(s)=\frac{m_{\mathrm{tot}}}{2} \cdot v(s)^{2},
$$

where $m_{\text {tot }}$ is the mass of the car, including its components' weight such as $m_{\mathrm{m}}, m_{\mathrm{gb}}$, and $m_{\mathrm{b}}$ for EM, transmission and battery, respectively. To preserve convexity, we relax the kinetic energy as

$$
E_{\mathrm{kin}}(s) \geq \frac{m_{\mathrm{tot}}}{2} \cdot v(s)^{2} .
$$

Concerning the scope of this study, we disregard the lower level longitudinal, lateral, and vertical vehicle dynamics and decide to model the vehicle as a point mass. Applying Newton's Second Law in space-domain to this point mass yields

$$
\frac{\mathrm{d}}{\mathrm{d} s} E_{\mathrm{kin}}(s)=F_{\mathrm{p}}(s)-F_{\mathrm{d}}(s)
$$

where $F_{\mathrm{p}}(s)$ is the propulsion force and $F_{\mathrm{d}}(s)$ is the drag force. The drag force is the sum of the aerodynamic drag force, the gravitational force, and the rolling resistance force:

$$
\begin{aligned}
& F_{\mathrm{d}}(s)=\frac{c_{\mathrm{d}} \cdot A_{\mathrm{f}} \cdot \rho}{m_{\mathrm{tot}}} \cdot E_{\mathrm{kin}}(s)+ \\
& m_{\mathrm{tot}} \cdot g \cdot\left(\sin (\theta(s))+c_{\mathrm{r}} \cdot \cos (\theta(s))\right),
\end{aligned}
$$

where $c_{\mathrm{d}}$ is the drag coefficient, $A_{\mathrm{f}}$ is the frontal area of the vehicle, $\rho$ is the density of air, $g$ is the earth's gravitational constant, $\theta(s)$ is the inclination of the track, and $c_{\mathrm{r}}$ is the rolling friction coefficient. The propulsive force at the wheels is equal to

$$
F_{\mathrm{p}}(s)= \begin{cases}\eta_{\mathrm{fd}} \cdot F_{\mathrm{gb}}(s)-F_{\mathrm{brk}}(s) & \text { if } F_{\mathrm{gb}}(s) \geq 0 \\ \frac{1}{\eta_{\mathrm{fd}}} \cdot F_{\mathrm{gb}}(s)-F_{\mathrm{brk}}(s) & \text { if } F_{\mathrm{gb}}(s)<0,\end{cases}
$$

where $\eta_{\mathrm{fd}}$ is the efficiency of the final drive, $F_{\mathrm{gb}}(s)$ is the force on the secondary axle of the transmission and $F_{\mathrm{brk}}(s)$ is the force excited by the mechanical brakes. Since $F_{\text {brk }}(s) \geq 0$, the propulsion force can be relaxed to

$$
\begin{aligned}
& F_{\mathrm{p}}(s) \leq \eta_{\mathrm{fd}} \cdot F_{\mathrm{gb}}(s) \\
& F_{\mathrm{p}}(s) \leq \frac{1}{\eta_{\mathrm{fd}}} \cdot F_{\mathrm{gb}}(s) .
\end{aligned}
$$

We condense the grip limitations as well as the lateral vehicular dynamics during cornering into the maximum kinetic energy constraint

$$
E_{\mathrm{kin}}(s) \leq E_{\mathrm{kin}, \max }(s)=\frac{m_{\mathrm{tot}}}{2} \cdot v_{\max }^{2}(s) .
$$

As mentioned at the beginning of this section, the maximum speed profile can be either pre-computed or measured, capturing the way the expert driver is feeling the car. While not being significantly affected by the energy management strategies, $v_{\max }(s)$ is strongly influenced by $m_{\text {tot }}$, which must be accounted for when pre-computing it, as shown in Appendix A. Finally, considering a free-flow racing lap, we enforce periodicity on the speed of the car with

$$
E_{\text {kin }}(0)=E_{\text {kin }}(S)
$$

\section{Transmission}

In this section, we further construct the minimum-lap-time control problem with convex constraints, modeling the two transmission technologies, an FGT and a CVT. For the former, we assume a constant transmission efficiency. For the latter, we derive a variable-efficiency model that depends on input speed, output speed, and input power. We describe a general transmission ratio as

$$
\gamma=\frac{\omega_{\text {in }}}{\omega_{\text {out }}}
$$

where $\omega_{\text {in }}$ and $\omega_{\text {out }}$ are equal to the rotational speed of the primary and secondary axle, respectively. Consequently, the speed of the motor is given by

$$
\omega_{\mathrm{m}}(s)=\gamma(s) \cdot \omega_{\mathrm{gb}}(s),
$$

where $\omega_{\mathrm{gb}}(s)=v(s) \cdot \frac{\gamma_{\mathrm{fd}}}{r_{\mathrm{w}}}$ is the output speed of the transmission, $\gamma_{\mathrm{fd}}$ is the fixed transmission ratio of the final drive, $r_{\mathrm{w}}$ is the radius of the wheels, and $\gamma(s)$ is the ratio of the transmission. For the transmission ratio, we define

$$
\gamma(s) \begin{cases}=\gamma_{1} & \text { if FGT } \\ \in\left[\gamma_{\min }, \gamma_{\max }\right] & \text { if CVT, }\end{cases}
$$

where $\gamma_{1}>0$ is the fixed ratio of the FGT, and $\gamma_{\min }>0$ and $\gamma_{\max }>0$ are the lower and upper limit of the CVT ratio, respectively. Considering a push-belt CVT, its operation is limited by a constant ratio coverage $c_{\gamma}=\frac{\gamma_{\max }}{\gamma_{\min }}$, which can be expressed in convex form as

$$
\gamma_{\min }=c_{\gamma} \cdot \gamma_{\max }
$$




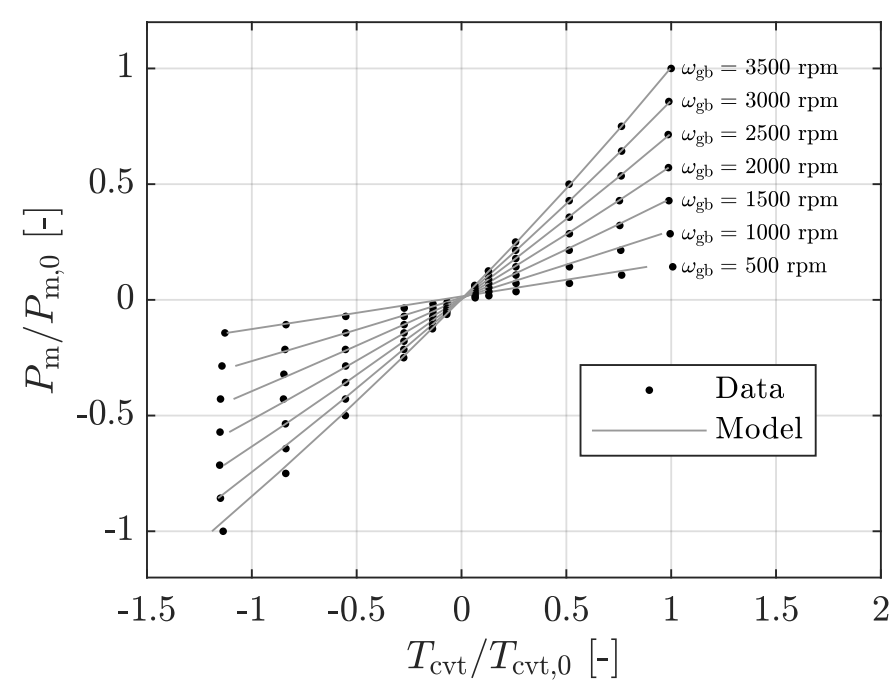

Fig. 2. A model of the CVT, dependent on the input speed, output speed, and input power. In this figure, the model is shown for a constant transmission ratio $\gamma=1$ and different levels of output speed. The normalized root-meansquare error (RMSE) of the full model is $0.25 \%$.

allowing us to optimize the ratio limits of the CVT within this constraint. Assuming a constant FGT efficiency $\eta_{\text {fgt }}$, we model and relax the transmission force similar to (7):

$$
\begin{aligned}
& F_{\mathrm{gb}}(s) \leq \eta_{\mathrm{fgt}} \cdot F_{\mathrm{m}}(s) \\
& F_{\mathrm{gb}}(s) \leq \frac{1}{\eta_{\mathrm{fgt}}} \cdot F_{\mathrm{m}}(s) .
\end{aligned}
$$

To capture the impact of the operating point on the efficiency of the CVT, the losses are fitted as a function of input speed, output speed, and input power. We can approximate the nonlinear model in a convex manner, using a quadratic approach. In that case, the losses of the CVT $P_{\mathrm{cvt} \text {,loss }}(s)$ are equal to

$$
P_{\mathrm{cvt}, \mathrm{loss}}(s)=x_{\mathrm{cvt}}(s)^{\top} Q_{\mathrm{cvt}} x_{\mathrm{cvt}}(s),
$$

where $x_{\mathrm{cvt}}(s)=\left[\begin{array}{llll}1 & \omega_{\mathrm{m}}(s) & \omega_{\mathrm{gb}}(s) & P_{\mathrm{m}}(s)\end{array}\right]^{\top}, P_{\mathrm{m}}(s)$ is the output power of the motor, and thus the input power of the transmission, and $Q_{\mathrm{cvt}}$ is a symmetric and positive semi-definite matrix. The values of $Q_{\text {cvt }}$ are determined using semi-definite programming solvers. The fitting results in a normalized root-mean-square error (RMSE) of $0.25 \%$. The loss power of the transmission can also be expressed by

$$
P_{\text {cvt }, \operatorname{loss}}(s)=P_{\mathrm{m}}(s)-P_{\mathrm{gb}}(s) .
$$

Fig. 2 shows the resulting CVT model for a certain fixed transmission ratio and different given levels of output speed. For reasons of confidentiality, sensitive data have been normalized. We relax constraint (14), substitute (13), and convert it to forces as

$$
\left(F_{\mathrm{m}}(s)-F_{\mathrm{gb}}(s)\right) \cdot \frac{\mathrm{d} t}{\mathrm{~d} s}(s) \geq y_{\mathrm{cvt}}(s)^{\top} Q_{\mathrm{cvt}} y_{\mathrm{cvt}}(s),
$$

where $y_{\mathrm{cvt}}(s)=\left[\begin{array}{llll}\frac{\mathrm{d} t}{\mathrm{~d} s}(s) & \gamma(s) \cdot \frac{\gamma_{\mathrm{fd}}}{r_{\mathrm{w}}} & \frac{\gamma_{\mathrm{fd}}}{r_{\mathrm{w}}} & F_{\mathrm{m}}(s)\end{array}\right]^{\top}$. Finally, we define

$$
z_{\mathrm{cvt}}(s)=C_{\mathrm{cvt}} y_{\mathrm{cvt}}(s)
$$

where $C_{\mathrm{cvt}}$ is the Cholesky factorization [43] of $Q_{\mathrm{cvt}}$, i.e., $Q_{\mathrm{cvt}}=C_{\mathrm{cvt}}^{\top} C_{\mathrm{cvt}}$. This way, we can write

$$
y_{\mathrm{cvt}}(s)^{\top} Q_{\mathrm{cvt}} y_{\mathrm{cvt}}(s)=z_{\mathrm{cvt}}(s)^{\top} z_{\mathrm{cvt}}(s) .
$$

After substituting (17) into (15), which results in a geometric mean expression, we can formulate the second order conic constraint

$$
\begin{aligned}
& \left(F_{\mathrm{m}}(s)-F_{\mathrm{gb}}(s)\right) \cdot \frac{1}{F_{0}}-\frac{\mathrm{d} t}{\mathrm{~d} s}(s) \cdot v_{0} \geq \\
& \qquad \begin{array}{l}
2 \cdot z_{\mathrm{cvt}}(s) \\
\left\|\left(F_{\mathrm{m}}(s)-F_{\mathrm{gb}}(s)\right) \cdot \frac{1}{F_{0}}+\frac{\mathrm{d} t}{\mathrm{~d} s}(s) \cdot v_{0}\right\|_{2}
\end{array},
\end{aligned}
$$

where $F_{0}=1 \mathrm{~N}$ is a normalization constant. This relaxed constraint will hold with equality in the case where an optimal solution is attained and the battery energy is limited [22]. A similar reasoning can be applied to the convex relaxations we will perform on the electric motor and battery models introduced below.

To be able to scale the size of the electric motor-explored in more detail in the subsequent section-we scale the losses of the CVT correspondingly, namely in torque direction, while the ratio coverage and the speed-dependent behavior do not change. A visualization of the scaling of the efficiency map is shown for the electric motor in Section II-D below. Finally, we scale the CVT mass $m_{\text {cvt }}$ linearly with the EM sizing factor $s_{\mathrm{m}}$. Hereby, the limit case of a sizing factor of 2 can be interpreted as two CVTs (and EMs) in parallel. The mass of the transmission is then equal to

$$
m_{\mathrm{gb}} \begin{cases}=m_{\mathrm{fgt}} & \text { if FGT } \\ =m_{\mathrm{cvt}} \cdot s_{\mathrm{m}} & \text { if CVT, }\end{cases}
$$

where $m_{\mathrm{fgt}}$ is the constant mass of the FGT.

\section{Electric Motor}

This section derives a convex power and speed-dependent model of the EM, which we append to our set of constraints. We fit the EM losses as a function of mechanical power and speed similarly as for the CVT in Section II-C above. Specifically, the losses of the $\operatorname{EM} P_{\mathrm{m}, \operatorname{loss}}(s)$ are modeled as

$$
P_{\mathrm{m}, \operatorname{loss}}(s)=x_{\mathrm{m}}(s)^{\top} Q_{\mathrm{m}} x_{\mathrm{m}}(s),
$$

where $x_{\mathrm{m}}(s)=\left[\begin{array}{lll}1 & \omega_{\mathrm{m}}(s) & P_{\mathrm{m}}(s)\end{array}\right]^{\top}$ and $Q_{\mathrm{m}}$ is a symmetric and positive semi-definite matrix. Again, the values of $Q_{\mathrm{m}}$ can be determined via semi-definite programming and result in a normalized RMSE value of $0.23 \%$. This way, the electrical power can be expressed by

$$
P_{\mathrm{m}, \operatorname{loss}}(s)=P_{\mathrm{dc}}(s)-P_{\mathrm{m}}(s) .
$$

Fig. 3 shows the resulting speed-dependent electric motor model. We relax, substitute, and convert the equation to forces to derive an expression for the losses

$$
\left(F_{\mathrm{dc}}(s)-F_{\mathrm{m}}(s)\right) \cdot \frac{\mathrm{d} t}{\mathrm{~d} s}(s) \geq y_{\mathrm{m}}(s)^{\top} Q_{\mathrm{m}} y_{\mathrm{m}}(s),
$$




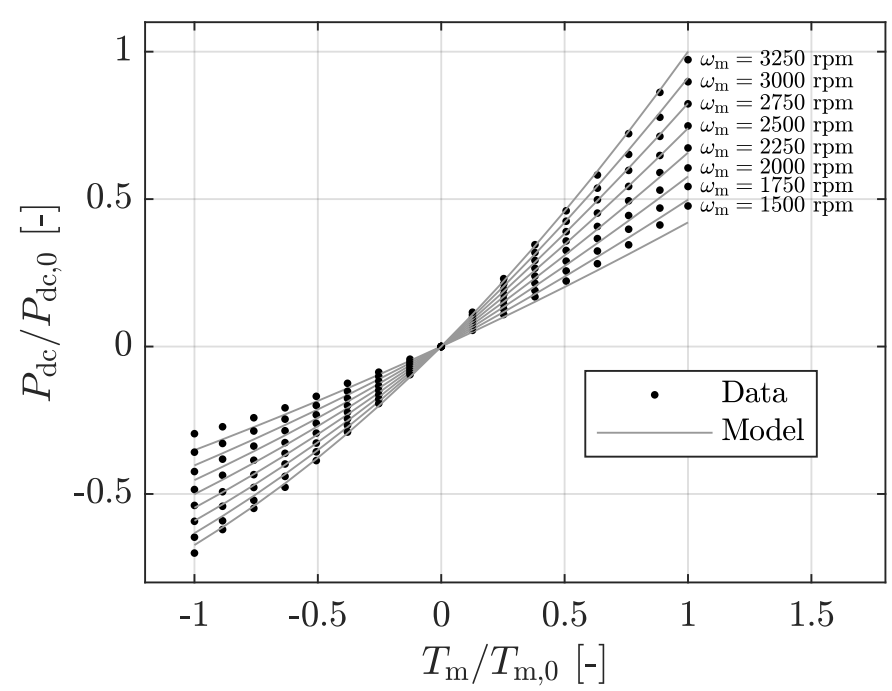

Fig. 3. An electric motor model, where the electrical power is presented as a function of mechanical torque for different motor speed levels. The normalized RMSE of the speed-dependent model is $0.23 \%$.

where $y_{\mathrm{m}}(s)=\left[\begin{array}{lll}\frac{\mathrm{d} t}{\mathrm{~d} s}(s) & \gamma(s) \cdot \frac{\gamma_{\mathrm{fd}}}{r_{\mathrm{w}}} & F_{\mathrm{m}}(s)\end{array}\right]^{\top}$ and $F_{\mathrm{m}}(s)$ is the force translation of mechanical motor power. We compute the Cholesky factorization as $Q_{\mathrm{m}}=C_{\mathrm{m}}^{\top} C_{\mathrm{m}}$ and define

$$
z_{\mathrm{m}}(s)=C_{\mathrm{m}} y_{\mathrm{m}}(s) \text {. }
$$

Using the same reasoning as for the CVT convex model, we can write $y_{\mathrm{m}}(s)^{\top} Q_{\mathrm{m}} y_{\mathrm{m}}(s)=z_{\mathrm{m}}(s)^{\top} z_{\mathrm{m}}(s)$ and substitute (21) into (20) to arrive at a geometric mean expression, which can be rewritten as a second order conic constraint

$$
\begin{aligned}
& \left(F_{\mathrm{dc}}(s)-F_{\mathrm{m}}(s)\right) \cdot \frac{1}{F_{0}}-\frac{\mathrm{d} t}{\mathrm{~d} s}(s) \cdot v_{0} \geq \\
& \qquad \begin{array}{l}
2 \cdot z_{\mathrm{m}}(s) \\
\left(F_{\mathrm{dc}}(s)-F_{\mathrm{m}}(s)\right) \cdot \frac{1}{F_{0}}+\frac{\mathrm{d} t}{\mathrm{~d} s}(s) \cdot v_{0}
\end{array} \|_{2} .
\end{aligned}
$$

The upper and lower bounds of the EM power can be divided into a maximum-torque and a maximum-power region. The maximum torque limit is approximated by the linear constraint

$$
P_{\mathrm{m}}(s) \in\left[-T_{\max } \cdot \omega_{\mathrm{m}}(s), T_{\max } \cdot \omega_{\mathrm{m}}(s)\right],
$$

where $T_{\max }$ is a constant maximum torque value. The maximum power region is captured by the affine function

$$
P_{\mathrm{m}}(s) \in\left[-c_{\mathrm{m}, 1} \cdot \omega_{\mathrm{m}}(s)-c_{\mathrm{m}, 2}, c_{\mathrm{m}, 1} \cdot \omega_{\mathrm{m}}(s)+c_{\mathrm{m}, 2}\right],
$$

where $c_{\mathrm{m}, 1} \leq 0$ and $c_{\mathrm{m}, 2} \geq 0$ are parameters subject to identification. The power and torque limits are visible in Fig. 4. The translation to forces results in

$$
F_{\mathrm{m}}(s) \in\left[-\frac{\gamma(s) \cdot T_{\max }}{r_{\mathrm{w}}}, \frac{\gamma(s) \cdot T_{\max }}{r_{\mathrm{w}}}\right]
$$

and

$$
F_{\mathrm{m}}(s) \in[-1,1] \cdot\left(\frac{c_{\mathrm{m}, 1} \cdot \gamma(s)}{r_{\mathrm{w}}}+c_{\mathrm{m}, 2} \cdot \frac{\mathrm{d} t}{\mathrm{~d} s}(s)\right) .
$$

To vary the size of the electric motor, we scale the minimum and maximum torque using a motor scaling factor defined as

$$
s_{\mathrm{m}}=\frac{T_{\max }}{\bar{T}_{\max }},
$$
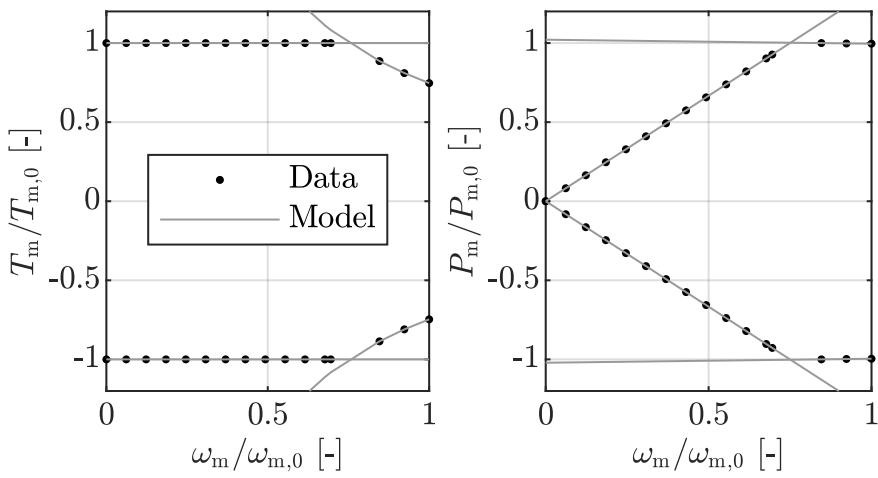

Fig. 4. The upper and lower bounds of the electric motor operating region.
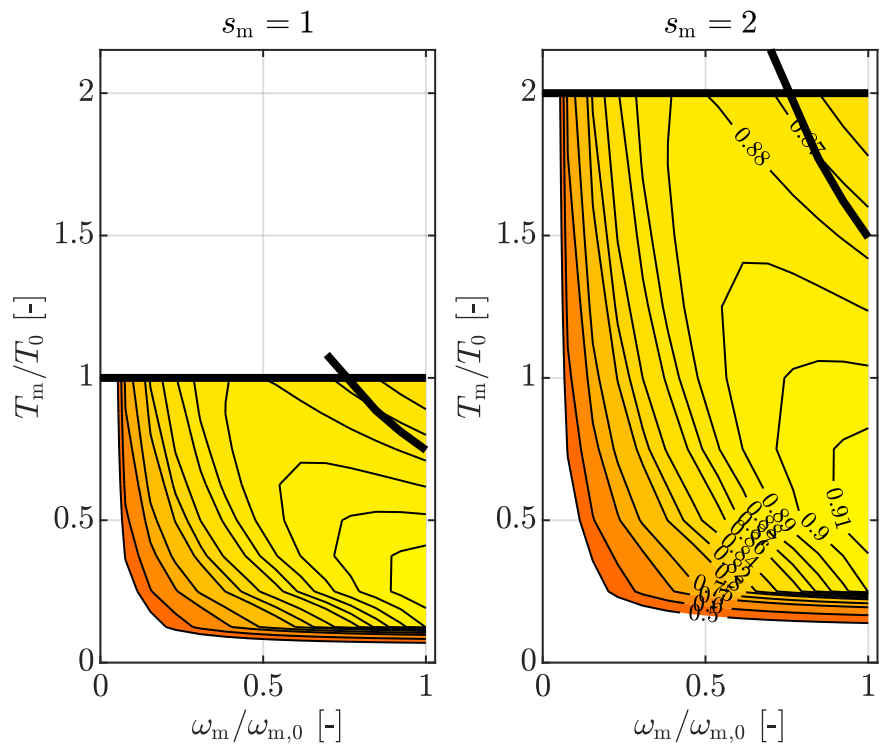

Fig. 5. The efficiency maps of an unscaled and $s_{\mathrm{m}}=2$ scaled EM model.

where $\bar{T}_{\max }$ is a base maximum torque value and where the notation $(\bar{\cdot})$ is used to indicate values corresponding to the original motor size. We scale the motor model parameters and mass $m_{\mathrm{m}}$ accordingly as

$$
\begin{aligned}
& c_{\mathrm{m}, i}=\bar{c}_{\mathrm{m}, i} \cdot s_{\mathrm{m}} \quad \forall i \in\{1,2\} \\
& m_{\mathrm{m}}=\bar{m}_{\mathrm{m}} \cdot s_{\mathrm{m}} .
\end{aligned}
$$

This way, the rated speed remains unchanged [37], [39]. We linearly scale the losses of the motor. To illustrate the method of scaling, Fig. 5 shows the efficiency maps and maximum torque and power functions for an unscaled motor and a motor scaled by $s_{\mathrm{m}}=2$.

Finally, the rotational speed of the motor cannot exceed the maximum speed $\omega_{\mathrm{m}, \max }$, which can be expressed as

$$
\gamma(s) \leq \omega_{\mathrm{m}, \max } \cdot r_{\mathrm{w}} \cdot \frac{\mathrm{d} t}{\mathrm{~d} s}(s) \cdot \frac{1}{\gamma_{\mathrm{fd}}} .
$$

\section{E. Battery Pack}

In this section, we expand the minimum-lap-time control problem constraints by deriving a model of the battery dy- 


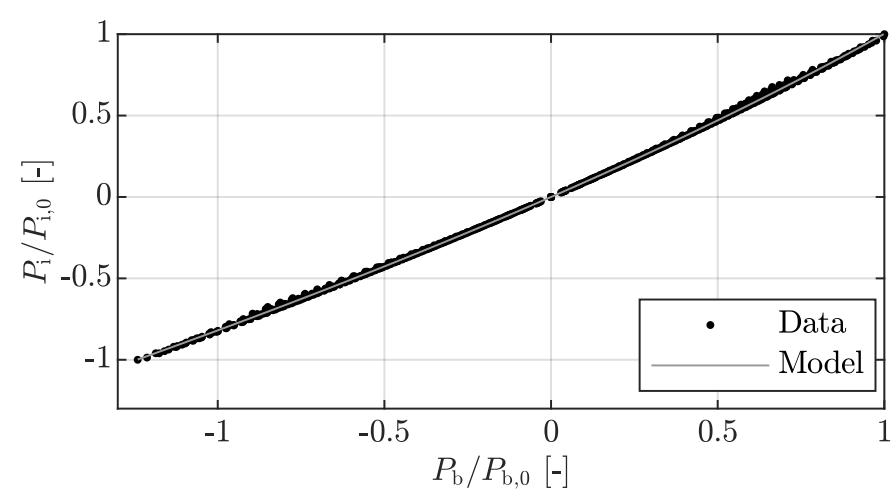

Fig. 6. A quadratic model of the losses in the battery pack was identified. The normalized RMSE is $0.69 \%$.

namics. The electric power at the terminals of the battery pack is

$$
P_{\mathrm{b}}(s)=P_{\mathrm{dc}}(s)+P_{\mathrm{aux}},
$$

where $P_{\text {aux }}$ is a constant auxiliary power. Converting this constraint to forces yields

$$
F_{\mathrm{b}}(s)=F_{\mathrm{dc}}(s)+P_{\mathrm{aux}} \cdot \frac{\mathrm{d} t}{\mathrm{~d} s}(s) .
$$

Leveraging a static circuit model [44], we approximate the internal battery power $P_{\mathrm{i}}(s)$ which causes the actual change in the battery state of energy $E_{\mathrm{b}}(s)$, to yield

$$
P_{\mathrm{i}}(s)=\alpha_{\mathrm{b}} \cdot P_{\mathrm{i}}(s)^{2}+P_{\mathrm{b}}(s),
$$

where the efficiency parameter $\alpha_{\mathrm{b}}$ (representing the average of the internal resistance divided by the open circuit voltage squared) is determined by a quadratic regression of the measurement data with a normalized RMSE of $0.69 \%$, as shown in Fig. 6. Similar as for the EM, relaxing this constraint and converting it to forces results in the second order conic constraint

$$
\begin{aligned}
& \frac{\mathrm{d} t}{\mathrm{~d} s}(s) \cdot v_{0}+\left(F_{\mathrm{i}}(s)-F_{\mathrm{b}}(s)\right) \cdot \frac{1}{F_{0}} \geq \\
& \left\|\begin{array}{c}
2 \cdot \sqrt{\alpha_{\mathrm{b}}} \cdot F_{\mathrm{i}}(s) \cdot \sqrt{\frac{v_{0}}{F_{0}}} \\
\frac{\mathrm{d} t}{\mathrm{~d} s}(s) \cdot v_{0}-\left(F_{\mathrm{i}}(s)+F_{\mathrm{b}}(s)\right) \cdot \frac{1}{F_{0}}
\end{array}\right\|_{2} .
\end{aligned}
$$

The battery energy consumption is modeled as

$$
\frac{\mathrm{d}}{\mathrm{d} s} \Delta E_{\mathrm{b}}(s)=F_{\mathrm{i}}(s)
$$

and is bounded as

$$
\begin{aligned}
& \Delta E_{\mathrm{b}}(0)=0 \\
& \Delta E_{\mathrm{b}}(S)=\Delta E_{\mathrm{b}, \max },
\end{aligned}
$$

where $\Delta E_{\mathrm{b}, \max }$ is the energy available to drive one lap. When scaling the battery size, we vary the amount of available battery energy by

$$
s_{\mathrm{b}}=\frac{\Delta E_{\mathrm{b}, \max }}{\Delta \bar{E}_{\mathrm{b}, \max }},
$$

where $\Delta \bar{E}_{\mathrm{b}, \max }$ is a nominal battery energy, resulting in a nominal battery pack mass $\bar{m}_{\mathrm{b}}$. Similarly, we scale the mass of the battery pack through

$$
m_{\mathrm{b}}=s_{\mathrm{b}} \cdot \bar{m}_{\mathrm{b}} \text {. }
$$

\section{F. Minimum-lap-time Optimization Problem}

Summarizing the objective function and the constraints derived in the previous sections, we can formulate the optimization problem to compute the achievable performance of the battery electric race car. Given an EM and battery size $s_{\mathrm{m}}$ and $s_{\mathrm{b}}$, the total mass of the vehicle results from (19), (26) and (33), which we use to compute the maximum speed profile as in A. We state the minimum-lap-time design and control problem for the state variables $x=\left(E_{\mathrm{kin}}, \Delta E_{\mathrm{b}}\right)$, control variables $u=\left(F_{\mathrm{m}}, \gamma\right)$ and design variables $p=\gamma_{1}$ and $p=\gamma_{\max }$ for the FGT and the CVT (given the constant ratio coverage $c_{\gamma}$ ), respectively, as follows:

Problem 1 (Minimum-lap-time Design and Control). The minimum-lap-time design and control strategies are the solution of

$$
\begin{aligned}
& \min \int_{0}^{S} \frac{\mathrm{d} t}{\mathrm{~d} s}(s) \mathrm{d} s \\
& \text { s.t. }(3)-(10),(21)-(25),(27)-(31) \\
& \text { and } \begin{cases}(12) & \text { if FGT } \\
(11),(16),(18) & \text { if CVT. }\end{cases}
\end{aligned}
$$

By inspecting the objective and constraints formulated in the previous sections, we observe that they are all individually convex. Therefore, we conclude that Problem 1 is fully convex and can be solved directly with second-order conic programming algorithms delivering a solution guaranteed to be globally optimal. The full second-order conic program formulation can be found in Appendix B.

\section{G. Discussion}

A few comments are in order. First, we assume that the cooling system is able to cope with the EM working at full power for a longer period of time. This assumption can be interpreted as a qualifying lap where the full capabilities of the EM can be exploited. Second, we scale the electric motor by scaling the efficiency and the mass linearly in the maximum torque. This scaling is in line with high-level modeling approaches and optimal sizing design problems [39]. More detailed scaling methods will be investigated in future research. Third, we assume the CVT to be able to cope with the torque requests, and scale it by scaling the losses in torque direction, corresponding to the sizing parameter of the electric motor. For instance, an EM scaling factor of 2 could be interpreted as the powertrain having two EMCVT sets in parallel, which can provide a qualitative insight into the behavior of a CVT in e-racing applications. We leave the study of more precise CVT scaling methods to future research. Finally, we assume that the battery is fully capable of delivering the electrical power that the electric motor is requesting and that the cooling system can cope with high charge and discharge rates. Again, this scenario can be interpreted as a qualifying lap.

\section{RESULTS}

This section presents numerical results, showcasing the optimization framework presented in Section II, i.e., the achievable 
lap times of different powertrain designs. First, we discuss the numerical solutions of the optimization problem, comparing the behavior of race cars equipped with an FGT and a CVT for a fixed-size powertrain. Second, we validate our methods via nonlinear simulations and verify that the relaxed constraints are indeed holding with equality. Third, we present a case study investigating the impact of the motor and battery size on the achievable lap time.

We discretize the continuous model presented in the previous section with the Euler Forward method with step-length $\Delta s=10 \mathrm{~m}$. We parse the problem as a second-order conic program with YALMIP [45] and solve it with MOSEK [46]. We perform our numerical studies on a Lenovo Yoga C940 laptop with an Intel Core i7-1065G7 $1.3 \mathrm{GHz}$ processor and $16 \mathrm{~GB}$ of RAM. Thereby, the computation time for one problem instance consists of about $6 \mathrm{~s}$ of parsing and $1 \mathrm{~s}$ of solving.

\section{A. Numerical Results}

In this section, we solve the posed minimum-lap-time control problem and compute the optimal state and input trajectories together with the transmission design for one race lap on the Le Mans racetrack. The mass of the vehicles under consideration are $1342 \mathrm{~kg}$ for the FGT and $1438 \mathrm{~kg}$ when using a CVT. We assume a final drive reduction efficiency equal to $\eta_{\mathrm{fd}}=99 \%$ and an FGT EM-to-wheel efficiency equal to $\eta_{\mathrm{fgt}}=98 \%$. Fig. 7 shows the optimal solution for both vehicles for $\Delta E_{\mathrm{b}, \max }=87 \mathrm{MJ}$, with the CVT-equipped car completing the lap in $220.6 \mathrm{~s}$ and $3.4 \mathrm{~s}$ faster than the car equipped with an FGT.

This lap time difference can mainly be explained by reasoning as follows: After the apex of relatively low-speed corners, the CVT car can lower the transmission ratio to instantly deploy the maximum EM power while keeping the EM speed constant, whereas the FGT car is operating at maximum torque in these sections of the track. Hence the CVT vehicle can accelerate faster, gain a time advantage, and increase the gap with respect to the FGT vehicle. Moreover, the variable transmission ratio allows a high-efficiency operation of the EM. We can also observe that the transmission ratio attained in the FGT solution limits the maximum speed of the car, since it is limited by the maximum rotational speed of the EM. On longer straight sections of the track, it can be noted that the FGT vehicle quickly accelerates to maximum speed and remains at that speed until braking into the next corner, whereas the CVT vehicle is continuously accelerating.

However, since the FGT has a higher transmission efficiency and a lower mass, it can achieve a higher cornering velocity and a higher acceleration at relatively high speeds, where the motor can operate at maximum power. This effect is visible in the final section of the track (from approximately $\mathrm{km} 11$ until the finish line), where the FGT vehicle outperforms the CVT in the combination of fast corners. This characteristic can also be observed in Fig. 9 which shows the acceleration capabilities of both cars for different vehicle velocities. It can be concluded that the CVT race car is capable of significantly higher accelerations, up to a velocity of approximately $205 \mathrm{~km} / \mathrm{h}$. Above this speed, until the FGT reaches its maximum speed, it
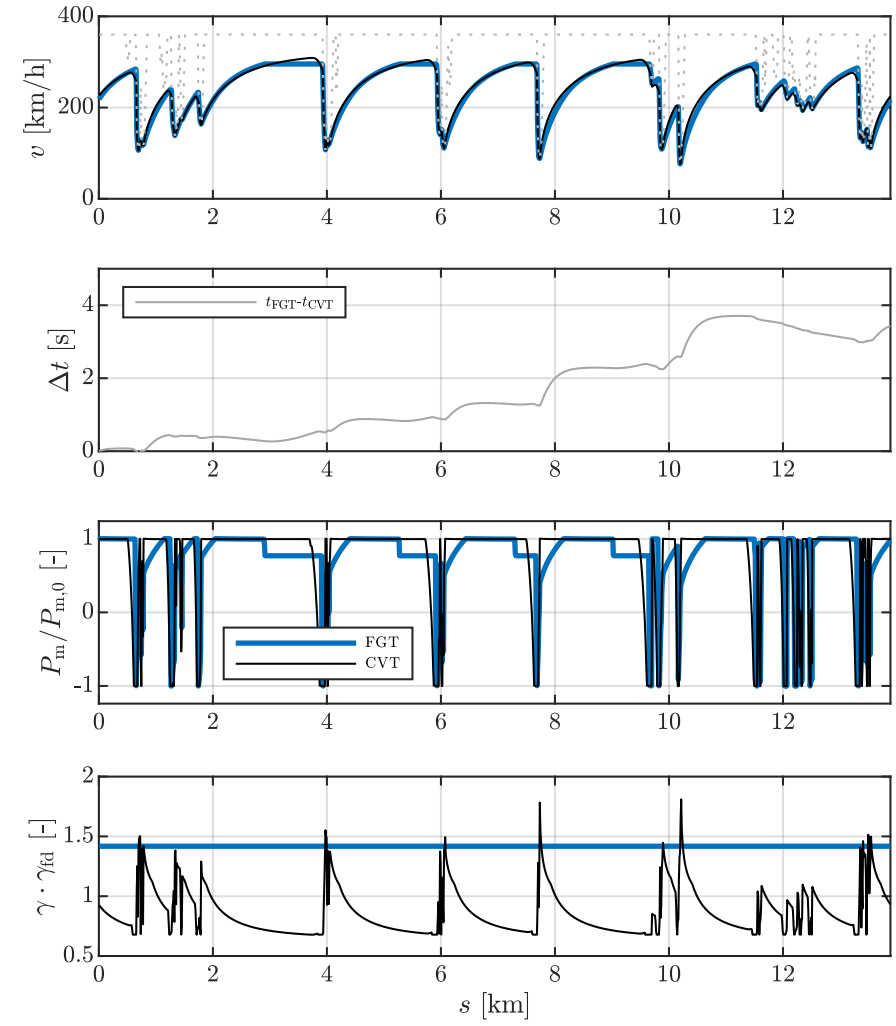

Fig. 7. Velocity, accumulated time difference, motor power, and ratio trajectory of both the FGT and the CVT vehicle.

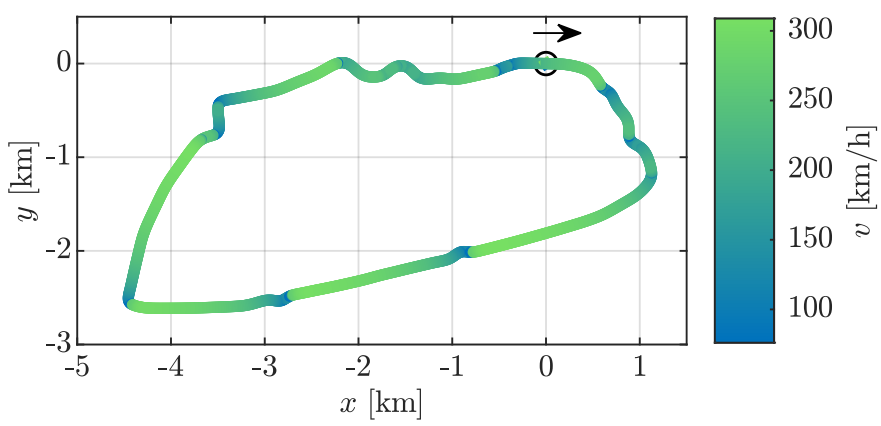

Fig. 8. The velocity of the CVT-equipped race car on the $x-y$ coordinates of the Le Mans race track. The circle indicates the location of start line and the arrow indicates the direction in which the lap is driven.

can achieve higher accelerations. This combination of factors leads to a faster lap time of the CVT race car.

Additionally, the velocity profile of the CVT-equipped car projected on the $x-y$ coordinates of the Le Mans track is presented in Fig. 8. This figure shows that the acceleration and braking phases coincide with the expectations based on the track layout and corner radii.

\section{B. Validation}

To validate our modeling and optimization framework, we feed the input trajectories attained by the minimum-lap-time control problem into a nonlinear simulator, consisting of the original data and efficiency maps of the CVT, electric motor and battery. For the FGT, we keep the efficiency $\eta_{\mathrm{fgt}}$ constant. Using the input trajectories from the optimal control problem 


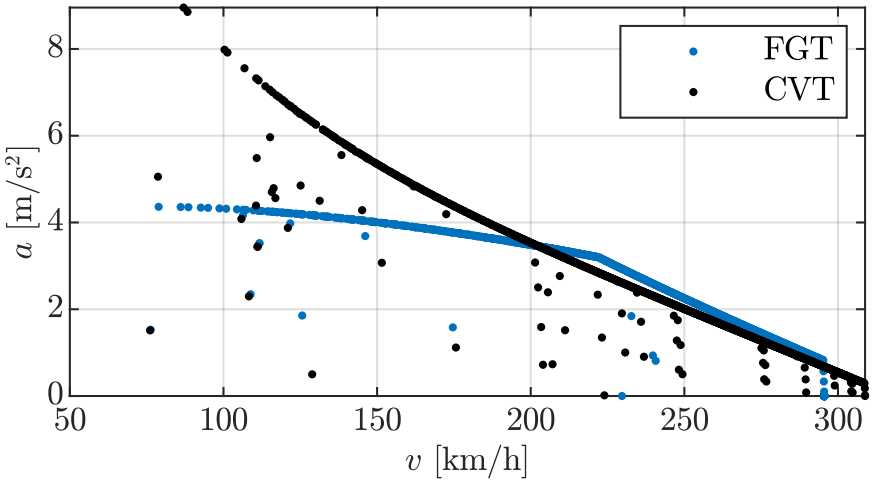

Fig. 9. Acceleration behavior of both the FGT and the CVT race cars as a function of speed.

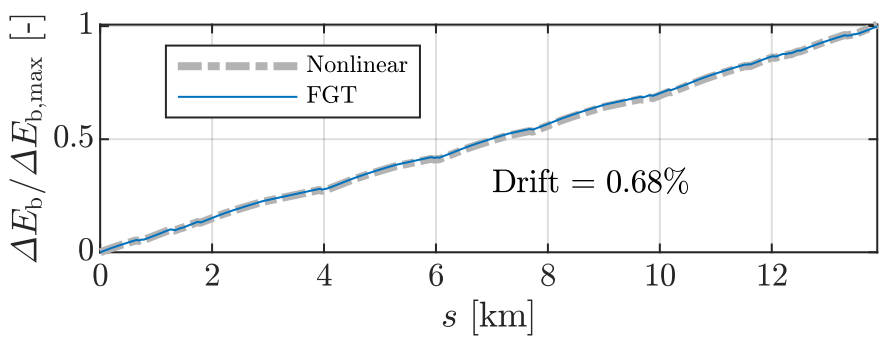

(a)

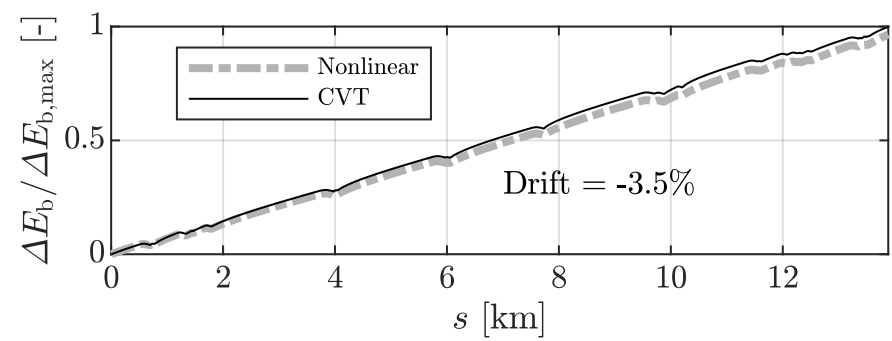

(b)

Fig. 10. The battery state of energy calculated through the convex and nonlinear models: (a) shows the nonlinear validation of the FGT model; and (b) shows the nonlinear validation of the variable-efficiency CVT model.

and the nonlinear simulator, we recalculate the battery energy consumption. Fig. 10 compares the resulting battery energy trajectory with the optimal battery trajectories for the FGT and the CVT car, indicating that our convex models can precisely capture the high-level powertrain dynamics with small deviations. Specifically, the battery energy drift of the FGT with respect to the nonlinear simulator is less than $1 \%$ (see Fig. 10a). The drift of the CVT vehicle (see Fig. 10b) is $-3.5 \%$, which is slightly less accurate due to the higher system complexity of the CVT.

Finally, we verify that all the relaxed constraints are active and hold with equality sign. As an example, Fig. 11 shows this feature for the lethargy and the kinetic energy secondorder conic constraints (3) and (4), respectively.

\section{Design Study}

The computational efficiency of the proposed method allows to perform extensive studies and gain qualitative and quantitative insights into the impact of different vehicle parameters on
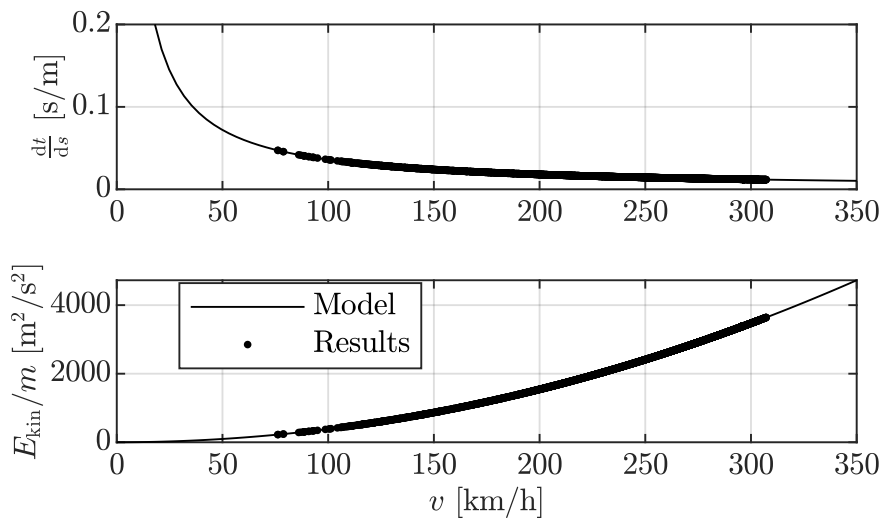

Fig. 11. Validation that the second order conic constraints of both velocity and kinetic energy are always active.

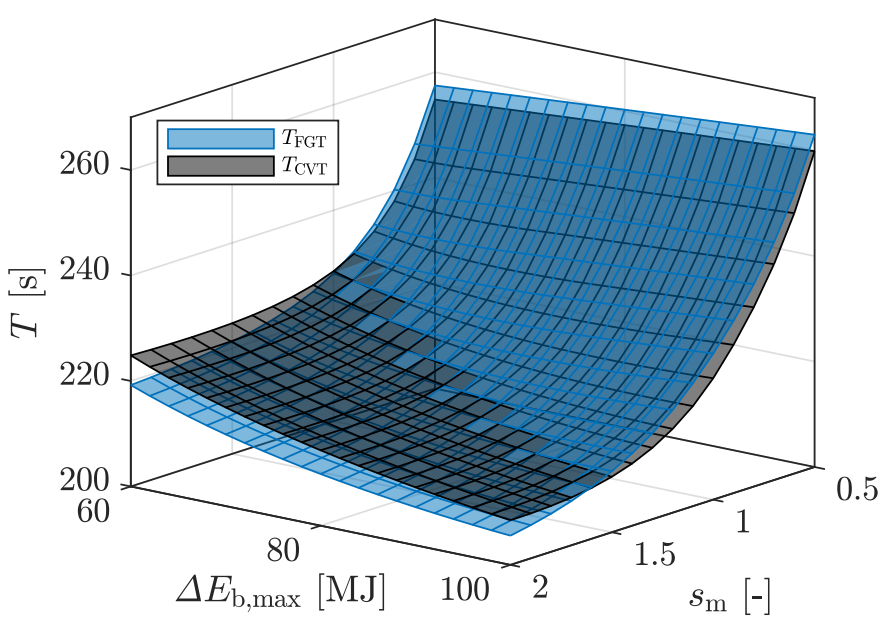

Fig. 12. Achievable lap times of the CVT and FGT defined for various motor and battery sizes.

the achievable lap time. To illustrate this, we vary the motor size with $s_{\mathrm{m}} \in[0.5,2]$ and the battery size as $\Delta E_{\mathrm{b}, \max } \in$ $[60,100]$ MJ. This size variation results in a grid of 336 points for which we compute the minimum lap time. The full computation lasted less than 1 hour, which is in line with the computational time measured for one single problem when including overhead. Critically, this kind of extensive search not only provides the globally optimal setup for the given scenario, but also offers a sensitivity analysis characterizing the impact of sizing variations on the achievable performance. The resulting lap times for both transmissions are shown in Fig. 12 and are compared in Fig. 13, leading to the conclusion that the CVT can outperform the FGT for a range of smaller motor sizes, since its potential to exploit maximum power at all speeds is enhanced for smaller motors. If the motor size is increased, the mass of the vehicle increases as well, and the lap time advantage of this aforementioned potential is reduced. Interestingly, increasing the battery size with a small motor results in a slower lap time, as the additional energy stored on-board cannot be deployed to counteract the higher mass of the car. Overall, the FGT vehicle seems to achieve the lowest possible lap time when both motor and battery size are maximized. We ascribe this difference to the significant 


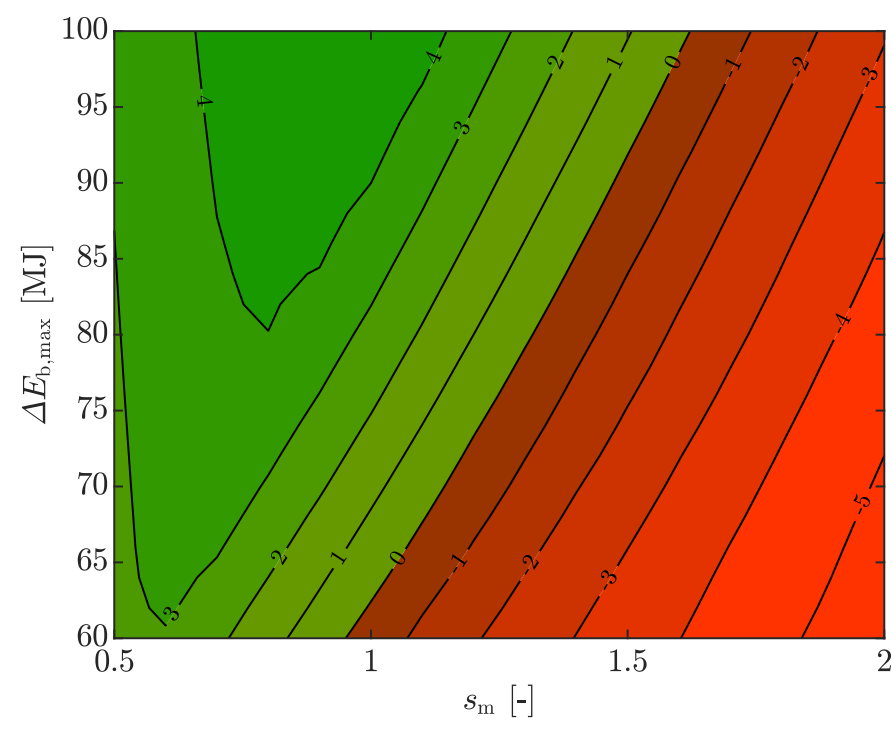

Fig. 13. Achievable lap time difference between CVT and FGT defined as $\Delta T=T_{\mathrm{FGT}}-T_{\mathrm{CVT}}$ for various motor and battery sizes.

increase in vehicular mass (almost 10\%) and to the slightly lower transmission efficiency resulting from the utilization of a CVT. However, we expect that near-future technological advancements could overcome this gap.

The upper plot of Fig. 14 shows similar results for different motor sizing and a constant $80 \mathrm{MJ}$ battery size. In particular, the FGT reaches the fastest lap time at the maximum motor size, whilst the CVT reaches a higher plateau at a slightly lower size. In both cases, increasing the motor size results in significant improvements in the range of tens of seconds. Similarly, the lower plot of Fig. 14 analyzes the impact of varying the battery size between 60 and $100 \mathrm{MJ}$ with a constant motor size of $s_{\mathrm{m}}=1$. Thereby, the optimal battery size for the FGT is smaller than for the CVT and results in a higher lap time. Specifically, increasing the battery size can improve the lap time, until reaching an energy value whereby the motor is able to operate at its limits during the complete lap. Any further battery increase only results in a higher vehicle mass without increasing the deployed propulsive power. Since the CVT-equipped car can request maximum motor power at every speed, it can deploy more EM power, hence a larger battery is advantageous. The impact of the battery size on the performance of a single vehicle is also significant. Finally, for both cases, the size of the battery pack shows a sensitivity with respect to the lap time in the range of several seconds.

\section{CONCLUSION}

This paper leveraged convex optimization methods to compute the minimum-lap-time control strategies for a battery electric race car. First, we showed that the minimum-laptime control problem can be framed in a fully convex form that can be rapidly solved in a few seconds using secondorder programming techniques and that the solution is guaranteed to be globally optimal. Second, the identified models showed a fitting error below $1 \%$, resulting in an overall battery consumption drift in the order of $4 \%$ when testing
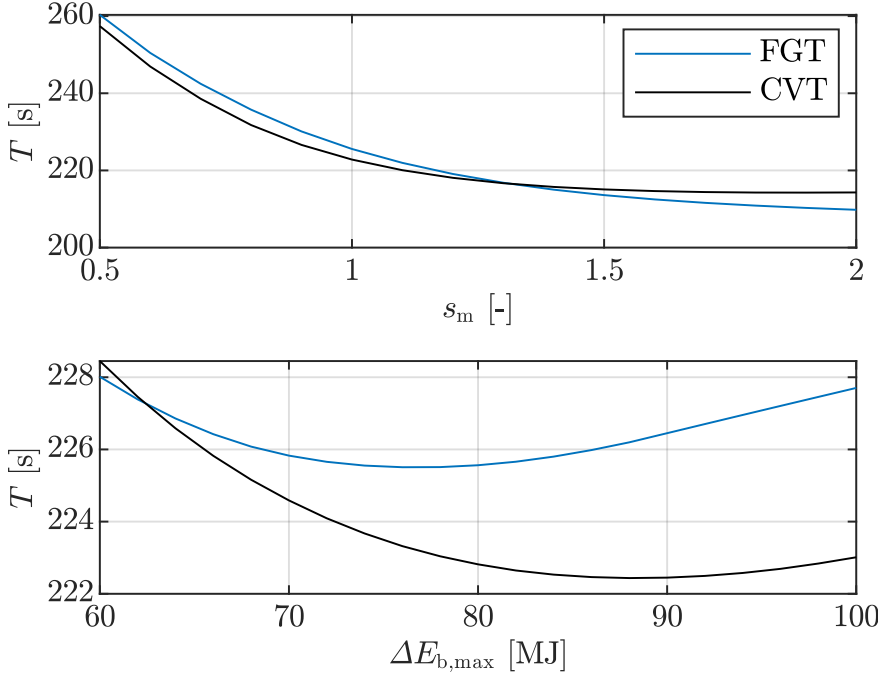

Fig. 14. Above, achievable lap time for varying battery sizes for the FGT and CVT vehicle, for fixed motor size $s_{\mathrm{m}}=1$. Below, achievable lap times for the FGT and CVT vehicle, for varying motor sizes and fixed available battery energy of $80 \mathrm{MJ}$.

our optimization results with nonlinear simulations. Third, our modeling framework explicitly captures the impact of speed on the motor efficiency, enabling us to design and compare two types of transmission, namely, a fixed-gear transmission (FGT) and a continuously variable transmission (CVT). In particular, our numerical studies for the Le Mans race track indicated that the lap time of a CVT-equipped electric race car can significantly outperform the one achievable with an FGT, as a CVT can counteract its higher mass with a more effective motor operation, although strongly dependent on the powertrain design choices. In fact, when studying the impact of the battery and motor size on the achievable lap time, we saw that using a CVT is beneficial for less powerful motors and higher battery consumptions, whilst the FGT reached the lowest possible lap time at maximum motor power and battery allowance.

This work calls for the following possible extensions: First, these promising preliminary results prompt a more detailed analysis based on high-fidelity models. Second, a more accurate insight in the achievable lap time can be obtained by incorporating optimal out-lap control, which is common in qualifying scenarios [24]. Third, these results suggest that research into finite-multispeed transmission technologies (e.g., a two-speed gearbox) would be of interest, since it would combine a higher efficiency with a lower mass. Fourth, this framework is modular and not limited to the proposed powertrain structure, as it can be readily applied to more complex topologies. Finally, we are interested in exploring the impact of the thermal behavior and heating effects of the electric motor and the battery on the optimal control strategy and the achievable lap time.

\section{ACKNOWLEDGMENT}

We are very grateful to Dr. I. New for proofreading this paper. Furthermore, we thank Dr. V. Varricchio for his advice. 


\section{APPENDIX A}

\section{MAXIMUM Velocity PROFILE}

This appendix presents methods to compute the maximum speed profile $v_{\max }(s)$. Considering data resulting from telemetry-measurements or computed in a high-fidelity simulator, we define the regions of the track where the driver is at full throttle to be power-limited, and grip-limited otherwise. The maximum velocity in the grip-limited regions is

$$
v_{\max }(s) \leq \sqrt{|R(s)| \cdot \frac{F_{\mathrm{y}, \max }(s)}{m_{\mathrm{tot}}}},
$$

where $R$ is the cornering radius and $F_{\mathrm{y}, \max }(s)$ is the maximum achievable lateral force. The latter is defined as

$$
F_{\mathrm{y}, \max }(s)=\mu \cdot F_{\mathrm{z}}(s),
$$

where $F_{\mathrm{z}}(s)$ is the normal force acting on the ground and $\mu$ is the stick friction coefficient. The normal force $F_{\mathrm{z}}(s)$ results from a sum of the vehicle's weight and the aerodynamic downforce $F_{\text {down }}(s)$ as

$$
F_{\mathrm{z}}(s)=m_{\text {tot }} \cdot g+F_{\text {down }}(s) .
$$

The aerodynamic downforce can be approximated as

$$
F_{\text {down }}(s)=\frac{1}{2} \cdot C_{1} \cdot \rho \cdot A_{\mathrm{f}} \cdot v(s)^{2},
$$

where $C_{1}$ is the vehicle's lift coefficient. Combining (34)-(37), the maximum velocity can be computed as

$$
v_{\max }(s) \leq \sqrt{\frac{|R(s)| \cdot \mu \cdot m_{\text {tot }} \cdot g}{m_{\text {tot }}-|R(s)| \cdot \mu \cdot \frac{1}{2} \cdot C_{\mathrm{l}} \cdot \rho \cdot A_{\mathrm{f}}}} .
$$

This equation is only valid in those parts of the track where the achievable lateral force is a limiting factor on the forward velocity. In other words, the velocity is only limited in the corners with a significantly small corner radius, where the aerodynamic downforce has an active influence on the achievable speed.

In the power-limited regions of the track, the maximum speed is set to a very high value and is connected to the grip-limited maximum speed by accounting for the vehicle approaching and launching from the grip-limited regions with a maximum deceleration and acceleration approximated by

$$
a_{\max }=\mu \cdot g .
$$

\section{APPENDIX B \\ SECOND Order Conic Program Formulation}

We state the full optimal control problem with state variables $x=\left(E_{\text {kin }}, \Delta E_{\mathrm{b}}\right)$, control variables $u=\left(F_{\mathrm{m}}, \gamma\right)$, design variables $p=\gamma_{1}$ and $p=\gamma_{\max }$ for the and FGT and CVT respectively, and lifting variables $\left(v, \frac{\mathrm{d} t}{\mathrm{~d} s}, F_{\mathrm{d}}, F_{\mathrm{gb}}, F_{\mathrm{dc}}, F_{\mathrm{b}}, F_{\mathrm{i}}\right)$ as

$$
\min \int_{0}^{S} \frac{\mathrm{d} t}{\mathrm{~d} s}(s) \mathrm{d} s
$$

subject to the dynamics

$$
\begin{aligned}
\frac{\mathrm{d}}{\mathrm{d} s} E_{\mathrm{kin}}(s) & =F_{\mathrm{p}}(s)-F_{\mathrm{d}}(s) \\
\frac{\mathrm{d}}{\mathrm{d} s} \Delta E_{\mathrm{b}}(s) & =F_{\mathrm{i}}(s),
\end{aligned}
$$

the state constraints

$$
\begin{aligned}
E_{\mathrm{kin}}(s) & \geq \frac{m_{\mathrm{tot}}}{2} \cdot v(s)^{2} \\
E_{\mathrm{kin}}(s) & \leq \frac{m_{\mathrm{tot}}}{2} \cdot v_{\text {max }}^{2}(s) \\
E_{\mathrm{kin}}(0) & =E_{\mathrm{kin}}(S) \\
\Delta E_{\mathrm{b}}(0) & =0 \\
\Delta E_{\mathrm{b}}(S) & =\Delta E_{\mathrm{b}, \max },
\end{aligned}
$$

the input constraints

$$
\begin{aligned}
& F_{\mathrm{m}}(s) \in\left[-\frac{\gamma(s) \cdot T_{\max }}{r_{\mathrm{w}}}, \frac{\gamma(s) \cdot T_{\max }}{r_{\mathrm{w}}}\right] \\
& F_{\mathrm{m}}(s) \in[-1,1] \cdot\left(\frac{c_{\mathrm{m}, 1} \cdot \gamma(s)}{r_{\mathrm{w}}}+c_{\mathrm{m}, 2} \cdot \frac{\mathrm{d} t}{\mathrm{~d} s}(s)\right) \\
& \gamma(s) \begin{cases}=\gamma_{1} & \text { if FGT } \\
\in\left[\gamma_{\min }, \gamma_{\max }\right] & \text { if CVT }\end{cases} \\
& \gamma(s) \leq \omega_{\mathrm{m}, \max } \cdot r_{\mathrm{w}} \cdot \frac{\mathrm{d} t}{\mathrm{~d} s}(s) \cdot \frac{1}{\gamma_{\mathrm{fd}}},
\end{aligned}
$$

the design constraint

$$
\gamma_{\min }=c_{\gamma} \cdot \gamma_{\max },
$$

and the physical constraints

$$
\begin{aligned}
& \frac{\mathrm{d} t}{\mathrm{~d} s}(s) \cdot v_{0}+v(s) \cdot \frac{1}{v_{0}} \geq\left\|\frac{\mathrm{d} t}{\mathrm{~d} s}(s) \cdot v_{0}-v(s) \cdot \frac{1}{v_{0}}\right\|_{2} \\
& F_{\mathrm{d}}(s)=\frac{c_{\mathrm{d}} \cdot A_{\mathrm{f}} \cdot \rho}{m_{\mathrm{tot}}} \cdot E_{\mathrm{kin}}(s)+ \\
& m_{\text {tot }} \cdot g \cdot\left(\sin (\theta(s))+c_{\mathrm{r}} \cdot \cos (\theta(s))\right) \\
& \begin{cases}F_{\mathrm{gb}}(s) \leq \eta_{\mathrm{fgt}} \cdot F_{\mathrm{m}}(s) & \\
F_{\mathrm{gb}}(s) \leq \frac{1}{\eta_{\mathrm{fgt}}} \cdot F_{\mathrm{m}}(s) & \text { if FGT } \\
\left(F_{\mathrm{m}}(s)-F_{\mathrm{gb}}(s)\right) \cdot \frac{1}{F_{0}}-\frac{\mathrm{d} t}{\mathrm{~d} s}(s) \cdot v_{0} \geq & \\
2 \cdot z_{\mathrm{cvt}}(s) & \\
\left\|\left(F_{\mathrm{m}}(s)-F_{\mathrm{gb}}(s)\right) \cdot \frac{1}{F_{0}}+\frac{\mathrm{d} t}{\mathrm{~d} s}(s) \cdot v_{0}\right\|_{2} & \text { if CVT }\end{cases} \\
& \left(F_{\mathrm{dc}}(s)-F_{\mathrm{m}}(s)\right) \cdot \frac{1}{F_{0}}-\frac{\mathrm{d} t}{\mathrm{~d} s}(s) \cdot v_{0} \geq \\
& \|\left(\begin{array}{c}
2 \cdot z_{\mathrm{m}}(s) \\
\left(F_{\mathrm{dc}}(s)-F_{\mathrm{m}}(s)\right) \cdot \frac{1}{F_{0}}+\frac{\mathrm{d} t}{\mathrm{~d} s}(s) \cdot v_{0}
\end{array} \|_{2}\right. \\
& F_{\mathrm{b}}(s)=F_{\mathrm{dc}}(s)+P_{\mathrm{aux}} \cdot \frac{\mathrm{d} t}{\mathrm{~d} s}(s) \\
& \frac{\mathrm{d} t}{\mathrm{~d} s}(s) \cdot v_{0}+\left(F_{\mathrm{i}}(s)-F_{\mathrm{b}}(s)\right) \cdot \frac{1}{F_{0}} \geq \\
& \left\|\begin{array}{c}
2 \cdot \sqrt{\alpha_{\mathrm{b}}} \cdot F_{\mathrm{i}}(s) \cdot \sqrt{\frac{v_{0}}{F_{0}}} \\
\frac{\mathrm{d} t}{\mathrm{~d} s}(s) \cdot v_{0}-\left(F_{\mathrm{i}}(s)+F_{\mathrm{b}}(s)\right) \cdot \frac{1}{F_{0}}
\end{array}\right\|_{2} .
\end{aligned}
$$




\section{REFERENCES}

[1] FIA. History - the formula e story. Available online at https://www. fiaformulae.com/en/discover/history.

[2] A. Sciarretta and L. Guzzella, "Control of hybrid electric vehicles," IEEE Control Systems Magazine, vol. 27, no. 2, pp. 60-70, 2007.

[3] V. Ngo, T. Hofman, M. Steinbuch, and A. Serrarens, "Optimal control of the gearshift command for hybrid electric vehicles," IEEE Transactions on Vehicular Technology, vol. 61, no. 8, pp. 3531-3543, Oct 2012.

[4] L. Tang, G. Rizzoni, and S. Onori, "Energy management strategy for hevs including battery life optimization," IEEE Transactions on Transportation Electrification, vol. 1, no. 3, pp. 211-222, 2015.

[5] J. Ritzmann, A. Christon, M. Salazar, and C. H. Onder, "Fuel-optimal power split and gear selection strategies for a hybrid electric vehicle," in SAE Int. Conf. on Engines \& Vehicles, 2019.

[6] L. V. Pérez and E. A. Pilotta, "Optimal power split in a hybrid electric vehicle using direct transcription of an optimal control problem," Mathematics and Computers in Simulation, vol. 79, no. 6, pp. 19591970, 2009.

[7] P. Elbert, S. Ebbesen, and L. Guzzella, "Implementation of dynamic programming for $n$-dimensional optimal control problems with final state constraints," IEEE Transactions on Control Systems Technology, vol. 21, no. 3, pp. 924-931, 2013.

[8] C. Lin, J. Kang, J. W. Grizzle, and H. Peng, "Energy management strategy for a parallel hybrid electric truck," in American Control Conference, 2001.

[9] P. Elbert, T. Nüesch, A. Ritter, N. Murgovski, and L. Guzzella, "Engine on/off control for the energy management of a serial hybrid electric bus via convex optimization," IEEE Transactions on Vehicular Technology, vol. 63, no. 8, pp. 3549-3559, 2014.

[10] N. Murgovski, L. Johannesson, X. Hu, B. Egardt, and J. Sjoberg, "Convex relaxations in the optimal control of electrified vehicles," in American Control Conference, 2015.

[11] N. Robuschi, M. Salazar, P. Duhr, F. Braghin, and C. H. Onder, "Minimum-fuel engine on/off control for the energy management of hybrid electric vehicles via iterative linear programming," in Symposium on Advances in Automotive Control, 2019.

[12] C. Balerna, N. Lanzetti, M. Salazar, A. Cerofolini, and C. H. Onder "Optimal low-level control strategies for a high performance hybrid electric power unit," Applied Energy, vol. 276, no. 115248, 2020.

[13] T. Nüesch, P. Elbert, M. Flankl, C. H. Onder, and L. Guzzella, "Convex optimization for the energy management of hybrid electric vehicles considering engine start and gearshift costs," Energies, vol. 7, no. 2, pp. 834-856, 2014.

[14] N. Robuschi, M. Salazar, N. Viscera, F. Braghin, and C. H. Onder, "Minimum-fuel energy management of a hybrid electric vehicle via iterative linear programming," IEEE Transactions on Vehicular Technology, vol. 69 , no. 12 , pp. $14575-14587,2020$

[15] H. Yu, F. Zhang, J. Xi, and D. Cao, "Mixed-integer optimal design and energy management of hybrid electric vehicles with automated manual transmissions," IEEE Transactions on Vehicular Technology, vol. 69 , no. 11 , pp. $12705-12715,2020$

[16] D. Casanova, "On minimum time vehicle manoeuvring: The theoretical optimal lap," Ph.D. dissertation, Cranfield University, 2000.

[17] R. Lot and S. Evangelou, "Lap time optimization of a sports series hybrid electric vehicle," in World Congress on Engineering, 2013.

[18] D. Limebeer, G. Perantoni, and A. Rao, "Optimal control of formula one car energy recovery systems," Int. Journal of Control, vol. 87, no. 10, pp. 2065-2080, 2014.

[19] D. J. Limebeer and A. V. Rao, "Faster, higher, and greener: Vehicular optimal control," IEEE Control Systems Magazine, vol. 35, no. 2, pp. 36-56, 2015.

[20] T. Herrmann, F. Passigato, J. Betz, and M. Lienkamp, "Minimum racetime planning-strategy for an autonomous electric racecar," in Proc. IEEE Int. Conf. on Intelligent Transportation Systems, 2020.

[21] X. Liu, A. Fotouhi, and D. Auger, "Optimal energy management for formula-e cars with regulatory limits and thermal constraints," Applied Energies, vol. 279, 2020

[22] S. Ebbesen, M. Salazar, P. Elbert, C. Bussi, and C. H. Onder, "Timeoptimal control strategies for a hybrid electric race car," IEEE Transactions on Control Systems Technology, vol. 26, no. 1, pp. 233-247, 2018.

[23] M. Salazar, P. Elbert, S. Ebbesen, C. Bussi, and C. H. Onder, "Timeoptimal control policy for a hybrid electric race car," IEEE Transactions on Control Systems Technology, vol. 25, no. 6, pp. 1921-1934, 2017.
[24] M. Salazar, P. Duhr, C. Balerna, L. Arzilli, and C. H. Onder, "Minimum lap time control of hybrid electric race cars in qualifying scenarios," IEEE Transactions on Vehicular Technology, vol. 68, no. 8, pp. 72967308, 2019.

[25] P. Duhr, G. Christodoulou, C. Balerna, M. Salazar, A. Cerofolini, and C. H. Onder, "Time-optimal gearshift and energy management strategies for a hybrid electric race car," Applied Energy, vol. 282, no. 115980, 2020.

[26] T. Hofman and C. Dai, "Energy efficiency analysis and comparison of transmission technologies for an electric vehicle," in IEEE Vehicle Power and Propulsion Conference, 2010.

[27] M. A. Kluger and D. M. Long, "An overview of current automatic, manual and continuously variable transmission efficiencies and their projected future improvements," in SAE World Congress, 1999.

[28] S. Ebbesen, C. Dönitz, and L. Guzzella, "Particle swarm optimization for hybrid electric drive-train sizing," International Journal of Vehicle Design, vol. 58, no. 2-4, pp. 181-199, 2012.

[29] S. Ebbesen, P. Elbert, and L. Guzzella, "Engine downsizing and electric hybridization under consideration of cost and drivability," Oil \& Gas Science and Technology-IFP Energies Nouvelles, vol. 68, no. 1, pp. 109-116, 2013

[30] F. J. R. Verbruggen, V. Rangarajan, and T. Hofman, "Powertrain design optimization for a battery electric heavy-duty truck," in American Control Conference, 2019.

[31] F. J. R. Verbruggen, E. Silvas, and T. Hofman, "Electric powertrain topology analysis and design for heavy-duty trucks," Energies, vol. 13, no. $10,2020$.

[32] C. A. Fahdzyana, M. Salazar, and T. Hofman, "Integrated plant and control design for a continuously variable transmission," IEEE Transactions on Vehicular Technology, 2021, in press.

[33] — , "A decomposed co-design strategy for continuously variable transmission design," in American Control Conference, 2021, in press.

[34] Y. Kim, H, D. G. Rideout, P. Y. Papalambros, and J. Stein, "Analytical target cascading in automotive vehicle design," ASME Journal of Mechanical Design, vol. 125, no. 3, pp. 481-489, 2003.

[35] M. Behtash and M. J. Alexander-Ramos, "A decomposition-based optimization algorithm for combined plant and control design of interconnected dynamic systems," ASME Journal of Mechanical Design, vol. 142 , no. 6, p. 061703,2020

[36] N. Murgovski, L. Johannesson, J. Sjöberg, and B. Egardt, "Component sizing of a plug-in hybrid electric powertrain via convex optimization," Mechatronics, vol. 22, no. 1, pp. 106-120, 2012.

[37] M. Pourabdollah, N. Murgovski, A. Grauers, and B. Egardt, "Optimal sizing of a parallel phev powertrain," IEEE Transactions on Vehicular Technology, vol. 62, no. 2, pp. 2469-2480, 2013.

[38] M. Pourabdollah, B. Egardt, N. Murgovski, and A. Grauers, "Convex optimization methods for powertrain sizing of electrified vehicles by using different levels of modelling details," IEEE Transactions on Vehicular Technology, vol. 67, no. 3, pp. 1881-1893, 2018.

[39] F. J. R. Verbruggen, M. Salazar, M. Pavone, and T. Hofman, "Joint design and control of electric vehicle propulsion systems," in European Control Conference, 2020.

[40] H. Yu, F. Castelli-Dezza, and F. Cheli, "Optimal powertrain design and control of a 2-iwd electric race car," in IEEE Int. Conf. of Electrical and Electronic Technologies for Automotive, 2017.

[41] H. Yu, F. Cheli, and F. Castelli-Dezza, "Optimal design and control of 4-iwd electric vehicles based on a 14-dof vehicle model," IEEE Transactions on Vehicular Technology, vol. 67, no. 11, pp. $10457-$ 10469,2018

[42] O. Borsboom, C. A. Fahdzyana, M. Salazar, and T. Hofman, "Timeoptimal control strategies for electric race cars for different transmission technologies," in IEEE Vehicle Power and Propulsion Conference, 2020.

[43] S. Boyd and L. Vandenberghe, Convex optimization. Cambridge Univ. Press, 2004.

[44] L. Guzzella and A. Sciarretta, Vehicle propulsion systems: Introduction to Modeling and Optimization, 2nd ed. Springer Berlin Heidelberg, 2007.

[45] J. Löfberg, "YALMIP : A toolbox for modeling and optimization in MATLAB," in IEEE Int. Symp. on Computer Aided Control Systems Design, 2004.

[46] M. ApS. (2017) MOSEK optimization software. Available at https:// mosek.com/. 


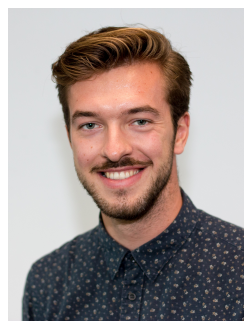

Olaf Borsboom obtained the B.Sc. degree and the M.Sc. degree in Automotive Technology with Great Appreciation from Eindhoven University of Technology (TU/e), The Netherlands, in 2017 and 2020, respectively. He is currently pursuing a Ph.D. degree at the Control Systems Technology section of TU/e. His main research interests include automotive technologies and optimization methods for powertrain design and control.

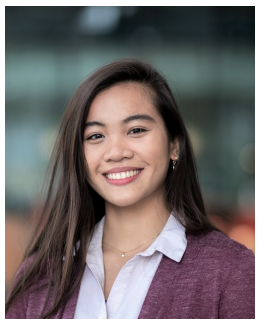

Chyannie Amarillio Fahdzyana obtained the B.Sc degree in Electrical Engineering from the University of Indonesia in 2015, and the M.Sc. degree in Automotive Technology from Eindhoven University of Technology, The Netherlands, in 2017. She is currently working towards a Ph.D. degree at the Control Technology section of the same university. Her main research interests include optimal system design, optimization and optimal control.

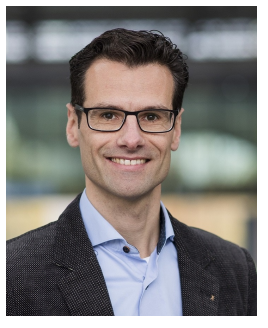

Theo Hofman is an Associate Professor in the Control Systems Technology section at Eindhoven University of Technology (TU/e). He received his M.Sc. (with honors) in 1999 and Ph.D. degree in 2007 in Mechanical Engineering from TU/e. From 1999 to 2003 , he was a researcher and project manager with the R\&D Department of Thales-Cryogenics B.V., Eindhoven. From 2003 to 2007, he was a scientific researcher at Drivetrain Innovations B.V., Eindhoven. From 2010 to 2017, he held the position of an Assistant Professor at TU/e. His research interests span system design optimization methods for complex dynamical engineering systems and discrete topology design using computational design synthesis.

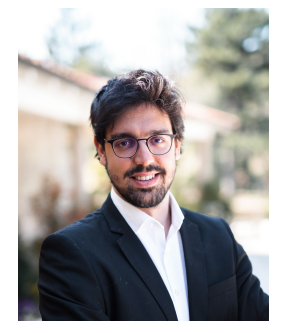

Mauro Salazar is an Assistant Professor in the Control Systems Technology section at Eindhoven University of Technology (TU/e). He received the $\mathrm{Ph} . \mathrm{D}$. degree in Mechanical Engineering from ETH Zürich in 2019. Before joining TU/e he was a Postdoctoral Scholar in the Autonomous Systems Lab at Stanford University. Dr. Salazar's research is at the interface of control theory and optimization, and is aimed at the development of a comprehensive set of tools for the design, the deployment and the operation of sustainable mobility systems. Both his Master thesis and PhD thesis were recognized with the ETH Medal, and he was granted the Best Student Paper award at the 2018 Intelligent Transportation Systems Conference. 